\title{
Perspective
}

\section{Innate immune responses to DNA viruses}

\author{
Ying Nie ${ }^{1,2}$, Yan-Yi Wang ${ }^{1 凶}$ \\ ${ }^{1}$ Wuhan Institute of Virology, Chinese Academy of Sciences, Wuhan 430071, China \\ ${ }^{2}$ Graduate University of Chinese Academy of Sciences, Beijing 100049, China \\ $\triangle$ Correspondence: wangyy@wh.iov.cn
}

The innate immune system acts as the first line of defense against pathogens, which is also essential for initiation of adaptive immunity. Innate immune responses are initiated by pattern-recognition receptors (PRRs), which recognize conserved molecular structures of pathogens called pathogen-associated molecular patterns (PAMPs). The infection of virus triggers a series of signaling events leading to transcriptional induction of type I interferons (IFNs) and proinflammatory cytokines. In recent years, the mechanisms of viral RNA recognition and RNA virus-triggered signaling pathways have been well studied. However, it remains unclear on how DNA virus infection is sensed by host cells and triggers the host antiviral defense. Although ten potential viral DNA sensors have been reported, none of them is validated as a generally used sensor for distinct DNA viruses in divergent cell types and animals. Here, we provide a summary and perspective on recent advances in innate immune responses to DNA viruses.

In the past few years, there has been great progress in the understanding of innate immune recognition of viral components and signaling pathways triggered by viral infection that leads to activation of transcription factors such as NF-KB and interferon regulatory factors (IRFs). As a result of innate immune responses, a variety of antiviral proteins are induced, including type I IFNs, proinflammatory cytokines, chemokines and other effectors. Such molecules are involved in direct inhibition of viral replication, elimination of viral components from infected cells or induction of apoptosis of infected cells. Furthermore, innate immune signals can activate adaptive immunity, therefore, are fundamental for host immune clearance of pathogens (Medzhitov, 2007; Palm and Medzhitov, 2009; Akira, 2011).

Upon infection, the innate immune system recognizes structurally conserved components of pathogens which are termed as pathogen-associ- ated molecular patterns (PAMPs) via different germline-encoded pattern-recognition receptors (PRRs). Several families of PRRs, such as Toll-like receptors (TLRs), RIG-I-like receptors (RLRs), NOD-like receptors (NLRs) and certain DNA sensors, have been identified (Kumar et al., 2011). Among the identified PRRs, it has been demonstrated that RNA viruses are mostly detected by TLR3 and RLRs. TLR3 is localized on the endosomal membrane of certain immune cells. It has been shown that TLR3 recognize viral double strand (ds) RNA and signals through a TIR domain containing adapter protein called TRIF/TICAM1. TRIF mediates the activation of transcription factors NF-KB and IRF3 via TRAF6-TAK1-IKK and TRAF3-TBK1 dependent pathways respectively (Kawai and Akira, 2009).

In contrast to TLR3 which is localized to the endosomal membrane in specific immune cells, the RLR family members RIG-I and MDA5 are cyto- plasmic proteins and expressed in most cell types. Therefore, RLRs play key roles in detecting invading and replicated viral RNAs in the cytoplasm in most cell types. Both RIG-I and MDA5 contain two CARD domains at their N-terminus and an RNA helicase domain at their $\mathrm{C}$-terminus. Although they share similar structural features, RIG-I and MDA5 sense different species of RNA viruses. For examples, it has been shown that picornaviruses, such as encephalomyocarditis virus (EMCV), are specifically recognized by MDA5, whereas some viruses of the paramyxovirus and rhabdovirus families are detected by RIG-I (Kato et al., 2006). The downstream signaling pathways mediated by RIG-I and MDA5 are mostly conserved. Upon recognition of viral RNAs through their C-terminal RNA helicase domains, RIG-I and MDA5 undergo conformational changes and are recruited to the downstream mitochondria-associated adaptor protein VISA (also called 
MAVS, IPS-1, Cardif) through their respective CARD domains (Xu et al., 2005; O'Neill and Bowie, 2010). VISA acts as a central platform and activates downstream TAK1-IKK $\beta$ and TBK1/IKKE kinases, leading to activation of NF-KB and IRF3 and induction of type I IFNs (Fitzgerald et al., 2003; Sharma et al., 2003; Kawai et al., 2005; Meylan et al., 2005; Seth et al., 2005).

While innate immune responses to RNA viruses have been extensively characterized, it is still unclear or controversial on how DNA viruses are sensed as well as the signaling mechanisms triggered by DNA virus infection. So far, several candidate viral DNA sensors and their signaling pathways have been reported, which will be discussed below.

\section{TLR9}

The TLR family consists of 10 and 12 members in humans and mice respectively. Among them, TLR9 is primarily expressed in $B$ cells and plasmacytoid dendritic cells ( $p D C s$ ) and recognizes CpG DNA which is commonly found in prokaryotes and viruses but not in vertebrates (Hemmi et al., 2000). Unprocessed TLR9 is localized to the endoplasmic reticulum (ER), and upon proteolysis, mature TLR9 is translocated to the endosomes (Latz et al., 2004). Studies have demonstrated that TLR9 is involved in recognition of hepatitis B virus (HBV), murine cytomegalovirus (MCMV) and Epstein-Barr virus (EBV) (Krug et al., 2004; Cui and Diao, 2010; Fiola et al., 2010). Upon recognition of cytoplasmic viral DNA, TLR9 recruits its adaptor protein MyD88 to initiate two distinct signaling pathways in pDCs. MyD88 interacts with IRF7, which is phosphorylated by the serine/threonine kinases IRAK-1 and IKKa. The phosphorylated IRF7 is translocated into the nucleus to regulate the transcription of type I IFN genes (Honda et al., 2004). MyD88 is also associated with TRAF6, which activates the IKK kinase complex through TAK1 (Konno et al., 2009). The activated IKK phosphorylates IKBa, leading to its degradation and subsequent activation of NF-KB. The activated NF-KB induces transcription of inflammatory cytokines (Akira and Takeda, 2004). It has been reported that the compartment in which TLR9 binds to its ligand is responsible for the bifurcation of TLR9 signaling (Sasai et al., 2010). Upon stimulation by viral nucleic acids, TLR9 is transported to the early endosomes to trigger the NF-KB activation pathway, and subsequently traffics to lysosome-related organelles (LRO) to induce type I IFNs (Kawai and Akira, 2011).

Although the endosomal localized TLR9 is capable of recognizing viral DNA, studies have demonstrated that herpes simplex virus-1 (HSV-1) infection, as well as transfected cytoplasmic DNAs, can induce the production of type I IFNs via a TLR9-independent pathway (Hochrein et al., 2004; Ishii and Akira, 2006; Stetson and Medzhitov, 2006). These studies suggest that other cellular DNA sensors exist for detection of DNA virus infection.

\section{DAI}

DAI, also called DLM-1/ZBP1, was the first molecule that has been suggested to function as a cytoplasmic DNA sensor (Takaoka et al., 2007). So far, three biological active double helical structures of DNA have been reported, including A-, B- and Z-form DNA. The most common and best-known DNA structure is the B-DNA which is characteristic of a right-handed double helix. Similarly, A-DNA shares the righthanded double helical structure, but is more compact. Z-DNA, in contrast, is typical of a left-handed double helical structure. DAl contains two Z-DNA binding domains at the $\mathrm{N}$-terminus and therefore is predicted to bind Z-DNA. Overexpression of DAl enhances the induction of type I IFNs by cytosolic DNA from different sources including bacterial, synthetic and mammalian DNA. Consistently, knockdown experiments using DAl specific RNAi suggest that DAl is important for type I IFN induction by different DNA sources as well as HSV infection. Interestingly, it has also been shown that DAI not only binds to Z-DNA but also to B-DNA, and its binding to $B-D N A$ induces the interaction between DAI and TBK1 or IRF3, resulting in the induction of type I IFNs (Takaoka et al., 2007). However, knockout studies reveal that, multiple cell types from DAl-deficient mice, including MEFs, bone marrow DCs and macrophages, respond normally to B-DNA-, plasmid DNA- as well as DNA virus-induced production of type I IFNs (Ishii et al., 2008). These studies suggest that DAI is not required for sensing of certain viral DNAs in the examined cell types.

Recently, DAI has been reported to be a partner of RIP3 and is essential for MCMV-induced cell death (Upton et al., 2012). Virus-induced cell death is an important mechanism of antiviral innate responses. However, further studies are required to fully elucidate the molecular mechanisms of DAI-RIP3 mediated cell death and its significance in antiviral response. For example, it is unclear whether the DNA-sensing activity of DAI is required for the induction of cell death and antiviral response.

\section{RNA POLYMERASE III}

RLRs, including RIG-I and MDA5, play important roles in the recognition of RNA viruses (Loo and Gale, 2011). Surprisingly, one study shows that the synthetic dsDNA poly (dA:dT) fails to induce IFN- $\beta$ in Huh-7.5.1 cell line harboring an inactive point mutation of RIG-I, suggesting that RIG-I is required for dsDNA-induced production of type I IFNs in these cells (Cheng et al., 2007). This result was surprising because it was well established that RIG-I is an RNA- instead of a DNA-binding protein. Later on, two groups identified RNA polymerase III as a cytosolic DNA sensor which subsequently activates the induction of type I IFNs through the RIG-I pathway (Ablasser et al., 2009; Chiu et al., 2009). Both studies demonstrate that, AT-rich dsDNA serves as a template which is converted into 
dsRNA with a 5'-triphosphoate moiety by RNA polymerase III. The transcript is a preferred ligand of RIG-I thus subsequently induces IFN- $\beta$ through the RIG-I pathway (Chiu et al., 2009). It has been shown Legionella pneumophila, HSV-1 and EBV all trigger the RNA polymerase III-mediated induction of type I IFNs (Chiu et al., 2009). As an important innate recognition mechanism, the RNA polymerase III pathway is targeted by certain pathogens. For example, it is blocked by vaccinia virus $E 3$ protein which binds to transcribed AU-rich RNA through its dsRNA-binding domain therefore prevents its recognition by RIG-I (Valentine and Smith, 2010).

Recognition of dsRNA intermediates produced by RNA polymerase III-mediated transcription of dsDNA by RIG-I shed new light on the mechanisms of innate immune detection of DNA viruses. However, redundancy still exists. It has been observed that, in human cells, non AT-rich dsDNA induces IFN- $\beta$ without the generation of an RNA intermediate (Ablasser et al., 2009), suggesting there must be other DNA sensors which directly recognize dsDNAs instead of the RNA intermediates. Moreover, studies have shown that RIG-I or VISA deficient cells and mice respond to poly $(\mathrm{dA}: \mathrm{dT})$ normally, suggesting the existence of other cytosolic DNA sensors in mice (Kumar et al., 2006; Sun et al., 2006).

\section{MITA}

MITA (Mediator of IRF3 Activation) (Zhong et al., 2008), also known as STING (Stimulator of Interferon Genes) (Ishikawa and Barber, 2008), was identified as an important adapter protein for virus-triggered type I IFN induction. MITA is localized on the outer-membrane of mitochondria, ER and the mitochondria-associated membrane (MAM) structure (Zhong et al., 2008). In certain cell types, MITA is recruited to VISA and RIG-I upon RNA virus infection. MITA can also recruit TBK1 and IRF3 to the complex, in which TBK1 phosphorylates IRF3
(Zhong et al., 2008).

Studies have demonstrated that IFN- $\beta$ production in response to the infection of HSV-1 or Listeria monocytogenes, or transfection of purified Escherichia coli DNA, calf thymus (CT) DNA, and ISD (interferon stimulatory DNA) is abolished in MITA-deficient mouse embryonic fibroblasts (MEFs) (Ishikawa et al., 2009), suggesting that MITA is pivotal in mediating DNAinduced innate immune responses. Recently, MITA was found to directly bind to cyclic diguanylate monophosphate (c-di-GMP), which functions as a secondary messenger in bacteria and triggers the production of type I IFNs (Burdette et al., 2011). Significantly, Goldenticket (Gt) mice, a mouse strain harboring a single nucleotide mutation (T596A) of Mita, do not express MITA protein (Sauer et al., 2011). These Gt mice fail to produce type I IFNs upon infection with Listeria monocytogenes, or treatment with c-di-GMP and c-diAMP in vivo. Macrophages isolated from $G t$ mice are unable to produce type I IFNs in response to Listeria monocytogenes infection as well as c-di-GMP and c-di-AMP in vitro (Sauer et al., 2011). Structural studies have demonstrated a direct interaction between the C-terminus of MITA and c-di-GMP (Ouyang et al., 2012; Shang et al., 2012; Shu et al., 2012; Yin et al., 2012). Upon binding to c-di-GMP, the c-di-GMP binding domain (CBD) of MITA forms a $V$-shaped dimer in which each arm of the " $V$ " is constituted of a monomer. The interface of the dimer which locates at the bottom of the " $\mathrm{V}$ " is the c-di-GMP binding site. A recent study has shown that the very C-terminal tail (CTT) of MITA is sufficient and required for IRF3 activation. Importantly, it has been shown that, under physiological conditions, the CTT of MITA interacts with the CBD intramolecularly and therefore results in an autoinhibition of MITA. Upon stimulation, c-diGMP displaces the CTT from the CBD thus results in the activation of the signaling cascades (Yin et al., 2012).

These studies have established that, in addition to functioning as a signaling adaptor, MITA also acts as a PRR and plays a pivotal role in sensing cytoplasmic nucleic acids.

\section{IFI16}

IFI16, a member of the HIN-200 protein family, contains a C-terminal HIN-200 domain for DNA binding and an N-terminal pyrin domain (PYD) for protein-protein interaction. It has been shown that IFI16 binds to both ss and ds DNAs (Unterholzner et al., 2010). IFI16 was originally reported as a nuclear protein because it contains a nuclear localization signal (NLS) (Briggs et al., 2001). However, additional studies have revealed that the subcellular localization of IFI16 differ in different cell types. In fact, IFI16 has been detected in the nucleus, cytoplasm or both in different cell types (Veeranki and Choubey, 2012). Interestingly, DNA binding of IFI16 leads to different signaling pathways and the subcellular localization of IFI16 is decisive on which pathway it activates. When localized in the cytoplasm, activated IFI16 recruits MITA, which leads to IRF3 and NF-KB activation as well as induction of IFN- $\beta$ in the macrophage cell line THP-1 (Unterholzner et al., 2010). Overexpression of IFI16 decreases the replication of HCMV (Gariano et al., 2012), whereas knockdown of IFI16 inhibits DNA- and HSV1-mediated activation of IRF3 and NF-kB (Unterholzner et al., 2010). When localized in the nucleus, IFI16 functions as a nuclear sensor to recruit ASC (apoptosis-associated speck-like protein containing a CARD) via their respective PYD domain and therefore induce the formation of inflammasomes (see AIM2 for further discussion of inflammasomes). The IFI16-inflammasome is initially formed in the nucleus and subsequently detected in the perinuclear area in response to KSHV (Kaposi sarcoma associated herpes virus) (Kerur et al., 2011). However, this study fails to detect an up-regulated IL-1 $\beta$ secretion following the activation of the nuclear IFI16-inflammasomes. Therefore, fur- 
ther studies are needed to understand the significance of the nuclear IFI16-inflammasomes. Interestingly, it has been reported that IFI16 suppresses the activation of caspase-1 triggered by the AIM2 or NLRP3 inflammasomes (Veeranki et al., 2011), whereas knockdown of AIM2 increases the basal levels of IFI16 (Duan et al., 2011). Therefore, AIM2 and IFI16 may function in a balanced way for eliminating pathogens and avoiding autoimmune diseases.

Recently, a study shows that the encapsidated viral DNA generated by HSV d109 infection of human foreskin fibroblasts (HFFs) is released into the nucleus and at where it is sensed by the nuclear IFI16, leading to the activation of MITA-IRF3 pathway. Intriguingly, IFI16 remains in the nucleus during the process, indicating that an unknown downstream protein may exit from the nucleus and signal to the cytoplasmic localized MITA (Orzalli et al., 2012). Further studies are required to test the model in which nuclear IFI16 activates the cytoplasmic MITA-IRF3 pathway.

\section{DDX41}

DDX41, a member of the DEXDc family of helicases, has been reported to act as a cytosolic sensor for DNA virus (Zhang et al., 2011; Stein and FalckPedersen, 2012). It has been shown that DDX41 is responsible for cytosolic DNA sensing in human mDCs (myeloid dendritic cells), BMDCs (bone marrow derived dendritic cells) and monocytes. DDX41 binds to poly(dA:dT) or HSV-1 DNA via its DEADc domain, resulting in its interaction with MITA and activation of TBK1 (Zhang et al., 2011). Knockdown of DDX41 impedes the production of type I IFNs and cytokines in response to foreign DNA (Zhang et al., 2011).

A recent study demonstrates that the bacterial secondary messengers c-di-GMP and c-di-AMP are directly recognized by DDX41 but not MITA (Parvatiyar et al., 2012). Knockdown of DDX41 in mouse or human cells inhibits the activation of MITA, TBK1 and
IRF3 in response to c-di-GMP or c-di-AMP. Upon recognition of the bacterial secondary messengers, DDX41 interacts with MITA to induce the expression of type I IFNs (Parvatiyar et al., 2012). Since MITA has been demonstrated as a sensor for c-diGMP and c-di-AMP, it is important to investigate the relationship of MITAand DDX41-mediated recognition of c-di-GMP and c-di-AMP.

\section{LRRFIP1}

LRRFIP1 was originally identified as a protein that interacts with the mammalian homolog of Drosophila flightless I (Fli-I) (Liu and Yin, 1998). It has been reported that LRRFIP1 is able to detect poly(l:C), poly(dA:dT) and poly(dG:dC), as well as the infection of vesicular stomatitis virus (VSV) and Listeria monocytogenes in macrophages. Moreover, knockdown of LRRFIP1 by RNAi inhibits VSV- and Listeria monocytogenes-induced production of IFN- $\beta$ (Yang et al., 2010). Upon recognition of dsRNA, B-DNA or Z-DNA in the cytoplasm, LRRFIP1 interacts with $\beta$-catenin and promotes the activation of $\beta$-catenin. The activated $\beta$-catenin binds to the C-terminal domain of IRF3 and recruits the acetyltransferase p300 to induce the expression of IFN- $\beta$ (Yang et al., 2010). This study suggests that LRRFIP1 may function as a candidate DNA sensor. However, more studies are needed to establish a general role for LRRFIP1 in innate immune recognition of DNA viruses in various cell types and in vivo.

\section{HMGBs}

High-mobility group box (HMGB) proteins, including HMGB1, 2 and 3 in mammals, are highly conserved. HMGBs contain two DNA binding domains. Whereas HMGB1 is ubiquitously and abundantly expressed, the expression of HMGB2 and HMGB3 are limited to lymphoid organs and hematopoietic stem cells respectively (Vaccari et al., 1998; Bianchi and Manfredi, 2007).

Recently, HMGBs have been re- ported to play a role in sensing nucleic acids. While HMGB1 and HMGB3 have been suggested to recognize both cytosolic DNAs and RNAs, HMGB2 only recognizes cytosolic DNAs (Yanai et al., 2009) HMGB1 deficiency results in reduced induction of type I IFNs in response to both DNA and RNA stimulations, whereas HMGB2 deficiency only affects DNAtriggered induction of type I IFNs in MEFs (Yanai et al., 2009). However, where and how HMGBs bind to viral nucleotides remains unclear. In addition, further studies are required for understanding HMGB-mediated downstream signaling pathways.

\section{LSm14A}

LSm14A, a member of the LSm family involved in RNA processing in the processing bodies (P-bodies), has recently been demonstrated as a sensor of viral nucleic acids in the early phase of viral infection (Li et al., 2012). LSm14A binds to synthetic poly(l:C), poly(dA: dT) or viral DNA. Knockdown of LSm14A by RNAi markedly reduces IFN- $\beta$ induction by SeV and HSV-1, suggesting LSm14A mediates innate immune responses to both RNA and DNA viruses (Li et al., 2012). Very interestingly, LSm14A is translocated from the P-bodies to peroxisomes upon infection by both RNA and DNA viruses. In the peroxisomes, LSm14A recruits MITA to induce IFN- $\beta$ in responding to DNA viruses, and recruits RIG-I and VISA to induce IFN- $\beta$ in responding to RNA viruses ( $\mathrm{Li}$ et al., 2012). Since some viral nucleic acid sensors are induced by viral infection, the constitutively expressed LSm14A may act as the earliest sensor of viral nucleic acids, which is critical for induction of other viral nucleic acid sensors and amplification of induction of antiviral genes. In light of this discovery, it has been widely observed that P-bodies are important cellular structures involved in viral life cycles (Beckham and Parker, 2008). For example, some viral RNAs and proteins accumulate in the P-bodies and/or 
stress granules, thus, the P-bodies and/or stress granules may be primary sites for initiation of cellular antiviral response. Interestingly, it has been demonstrated that some well-known antiviral proteins, such as protein kinase $R$ (PKR) and APOBEC3 proteins, are enriched at the P-bodies during viral infection (Bechham and Parker, 2008). Interestingly, a recent study shows that nonstructural protein (NS1) of influenza A virus inhibits cellular antiviral response by targeting the Pbody-localized LSm14A/RAP55 (Mok et al., 2012). Taken together, these findings point to a critical role of LSm14A as well as the P-bodies in cellular antiviral response.

\section{AIM2 AND NLRP3}

In addition to the activation of TBK1 and IRF3, which results in the induction of type I IFNs, innate recognition of viral DNA also activates pathways leading to the assembly of inflammasomes, which, via caspase-1, result in the cleavage and maturation of certain proinflammatory cytokines such as IL-1 $\beta$ and IL-18 (Petrilli et al., 2007; Muruve et al., 2008).

So far, four types of inflammasomes have been identified, including the NLRP1-, NLRP3-, IPAF-, and AIM2inflammasomes (Kumar et al., 2012). NLRP1- and IPAF-inflammasomes mainly function against bacterial infection and therefore will not be discussed here.

AIM2 (absent in melanoma 2), a member of the HIN-200 family, was reported to be a regulator of DNAmediated inflammatory responses (Burckstummer et al., 2009; Fernandes-Alnemri et al., 2009; Hornung et al., 2009; Rathinam et al., 2010). AIM2 contains a conserved DNA binding domain called HIN-200 domain at its C-terminus and a PYD domain responsible for protein-protein interaction at its $\mathrm{N}$-terminus. It has been reported that AIM2 directly senses dsDNA from different sources, including mammalian, synthetic, bacterial, and viral DNA, in the cytoplasm and subsequently re- cruits the adaptor protein ASC via their respective $P Y D$. ASC interacts with pro-caspase-1 via their respective CARD domain. Pro-caspase- 1 is activated in this complex, leading to the processing and maturation of inflammatory cytokines, such as IL-1 $\beta$ and IL-18 (Hornung et al., 2009). Such AIM2-ASC-Caspase-1 complex is called AIM2-inflammasome. Further studies reveal that, upon DNA binding, AIM2 undergoes oligomerization that is important for the assembly of AIM2inflammasomes and caspase-1 activation (Fernandes-Alnemri et al., 2009). Intriguingly, it has also been found that, binding of dsDNA to AIM2 induces pyroptosis, a type of proinflammatory cell death that is different from apoptosis mediated by caspase- 3 activation (Fernandes-Alnemri et al., 2009; Wenzel et al., 2012). Since the expression of AIM2 is induced by IFNs (Landolfo et al., 1998), it is likely that, upon DNA virus infection, the host cells utilize both the TBK1-IRF3 and AIM2-inflammasome pathways to ensure a fulfilled innate response against viral infection.

Gene knockout in mice has shown that AIM2 is essential for the recognition of mouse MCMV or Listeria monocytogenes infection in both DCs and macrophages (Rathinam et al., 2010). In addition, AIM2-deficient mice are more susceptible to Francisella tularensis infection with higher mortality compared to wild-type mice (Fernandes-Alnemri et al., 2010).

In addition to AIM2, NLRP3 has also been reported to recognize adenoviral DNA and subsequently form the NLRP3-ASC-caspase-1 inflammasomes for IL-1 $\beta$ production (Muruve et al., 2008). However, further studies are required to determine whether NLRP3 and AIM2 function redundantly or distinctly.

\section{PERSPECTIVE}

So far, at least ten proteins have been reported to sense viral DNA. The signaling events mediated by the candidate viral DNA sensors have also been investigated (Table 1). The functions of these viral DNA sensors are distinctly regulated at several levels. Firstly, these sensors have different cellular expression profiles. For example, TLR9 is primarily expressed in certain immune cells such as pDCs (Wagner, 2004); HMGB2 is specifically expressed in lymphoid organs (Ronfani et al., 2001); DDX41 acts as a intracellular DNA sensor in mDCs, BMDCs and monocytes (Zhang et al., 2011); whereas the other DNA sensors, including MITA and RNA polymerase III, are expressed in most cell types. Moreover, even if ubiquitously expressed, such as RNA polymerase III, a sensor might have different functional significance in different cell types against different types of viruses. Second, the DNA sensors are located at distinct subcellular organelles or structures. For example, it has been shown that mature TLR9 is localized at the endosomes (Latz et al., 2004). LSm14A is localized at the P-bodies and is translocated to peroxisomes after viral infection. It has been hypothesized that LSm14A recognizes viral DNA at the P-bodies and then signals downstream via interacting with RIG-I-VISA or MITA in peroxisomes (Li et al., 2012). IFI16 has different signaling activities depending on its cellular localizations (Veeranki and Choubey, 2012). Therefore, host cells may have different sensors functioning at different subcellular compartments to provide multiple protections. Third, utilization of viral DNA sensors may be temporally regulated. It is possible that the affinity to viral DNA and abundance of the sensors in a cell dictates temporal utilization of the different viral DNA sensors. Finally, it is possible that distinct viral DNAs are preferentially recognized by different sensors and this preference may change among different species.

Although extensive studies have established the candidacies of the above mentioned proteins in recognition of viral DNAs, it is obvious that more rigorous experiments are needed to validate these proteins as bona fide 
Table 1. Candidate sensors for viral and other foreign DNAs

\begin{tabular}{|c|c|c|c|c|c|}
\hline DNA sensors & Ligands & Ligand binding domain & Expression profile & Cellular localization & Signaling \\
\hline TLR9 & $\begin{array}{l}\text { CpG DNA } \\
\text { HBV, MCMV, EBV }\end{array}$ & LRR & $\mathrm{pDCs}$ & Endosomes & $\begin{array}{l}\text { MyD88- IRF7 } \\
\text { MyD88- TAK1 }\end{array}$ \\
\hline DAl & $\begin{array}{l}\text { D-DNA } \\
\text { B-DNA } \\
\text { HSV-1 } \\
\text { MCMV }\end{array}$ & $Z \alpha$ and $Z \beta$ & Most cell types & Cytoplasm & $\begin{array}{l}\text { TBK1-IRF3 } \\
\text { RIP3 }\end{array}$ \\
\hline RNA Pol III & $\begin{array}{l}\text { dsDNA } \\
\text { HSV-1, EBV }\end{array}$ & Unknown & Most cell types & Cytoplasm & RIG-I-VISA-IRF3 \\
\hline MITA & c-di-GMP, c-di-AMP & CBD & Most cell types & Mitochondria, ER, MAM & TBK1-IRF3 \\
\hline IFI16 & $\begin{array}{l}\text { dsDNA } \\
\text { ssDNA } \\
\text { VACV, HSV-1, KSHV }\end{array}$ & HIN-200 & Most cell types & $\begin{array}{l}\text { Nucleus } \\
\text { Cytoplasm }\end{array}$ & $\begin{array}{l}\text { MITA-IRF3 } \\
\text { ASC-caspase-1 }\end{array}$ \\
\hline DDX41 & $\begin{array}{l}\text { dsDNA } \\
\text { c-di-GMP } \\
\text { HSV-1 }\end{array}$ & DEADc & $\begin{array}{l}\mathrm{mDCs} \\
\text { BMDCs }\end{array}$ & Cytoplasm & MITA-TBK1-IRF3 \\
\hline LRRFIP1 & $\begin{array}{l}\text { RNA } \\
\text { DNA }\end{array}$ & Unknown & Most cell types & Cytoplasm & $\beta$-catenin-IRF3 \\
\hline HMGB1 & $\begin{array}{l}\text { RNA } \\
\text { DNA } \\
\text { HSV-1 }\end{array}$ & Unknown & Most cell types & $\begin{array}{l}\text { Nucleus } \\
\text { Cytoplasm }\end{array}$ & Unknown \\
\hline HMGB2 & $\begin{array}{l}\text { DNA } \\
\text { HSV-1 }\end{array}$ & & Lymphoid organs & & \\
\hline HMGB3 & $\begin{array}{l}\text { RNA } \\
\text { DNA } \\
\text { HSV-1 }\end{array}$ & & $\begin{array}{l}\text { Hematopoitic } \\
\text { cells }\end{array}$ & & \\
\hline LSm14A & $\begin{array}{l}\text { RNA } \\
\text { DNA } \\
\text { HSV-1 }\end{array}$ & Unknown & Most cell types & P-bodies peroxisomes & $\begin{array}{l}\text { RIG-I-VISA } \\
\text { MITA }\end{array}$ \\
\hline AIM2 & $\begin{array}{l}\text { DNA } \\
\text { MCMV }\end{array}$ & $\mathrm{HIN}-200$ & Most cell types & Cytoplasm & ASC-caspase-1 \\
\hline NALP3 & $\begin{array}{l}\text { DNA } \\
\text { AdV }\end{array}$ & LRR & Most cell types & Cytoplasm & ASC-caspase-1 \\
\hline
\end{tabular}

viral DNA sensors. For examples, extensive biochemical experiments are needed to define the binding affinities and specificities of the candidate sensors to various viral DNAs. It is also important to extensively investigate the immune responses to infection by divergent types of viruses in knockout cells and mice of the candidate sensors. Although considerable advances have been made in the field of innate immune responses to DNA viruses, a master sensor to viral DNA still waits to be identified or validated.

Moreover, we also know little about the signaling mechanisms as well as their potential crosstalk of the candidate viral DNA sensors. Further investigation and understanding of these questions will certainly shed light on how the host responds to infection of DNA viruses and help to develop strategies to prevent and treat infectious diseases caused by these pathogens.

\section{ACKNOWLEDGMENTS}

Work in the authors' laboratory is supported by grants from the National Natural Science Foundation of China (Grant Nos. 31000639 and 31170792).

\section{REFERENCES}

Ablasser, A., Bauernfeind, F., Hartmann, G., Latz, E., Fitzgerald, K.A., et al. (2009). Nat Immunol 10, 1065-1072.

Akira, S. (2011). Philos Trans R Soc Lond B Biol Sci 366, 2748-2755.

Akira, S., and Takeda, K. (2004). Nat Rev Immunol 4, 499-511.

Bianchi, M.E., and Manfredi, A.A. (2007). Immunol Rev 220, 35-46.

Briggs, L.J., Johnstone, R.W., Elliot, R.M., Xiao, C.Y., Dawson, M., et al. (2001). Biochem J 353, 69-77.

Burckstummer, T., Baumann, C., Bluml, S., Dixit, E., Durnberger, G., et al. (2009). Nat Immunol 10, 266-272.

Burdette, D.L., Monroe, K.M., Sotelo-Troha, K., Iwig, J.S., Eckert, B., et al. (2011).
Nature 478, 515-518.

Cheng, G., Zhong, J., Chung, J., and Chisari, F.V. (2007). Proc Natl Acad Sci U S A 104, 9035-9040.

Chiu, Y.H., Macmillan, J.B., and Chen, Z.J. (2009). Cell 138, 576-591.

Cui, G.Y., and Diao, H.Y. (2010). Hepatobiliary Pancreat Dis Int 9, 584-592.

Duan, X., Ponomareva, L., Veeranki, S., Panchanathan, R., Dickerson, E., et al. (2011). Mol Cancer Res 9, 589-602.

Fernandes-Alnemri, T., Yu, J.W., Datta, P., $\mathrm{Wu}$, J., and Alnemri, E.S. (2009). Nature 458, 509-513.

Fernandes-Alnemri, T., Yu, J.W., Juliana, C., Solorzano, L., Kang, S., et al. (2010). Nat Immunol 11, 385-393.

Fiola, S., Gosselin, D., Takada, K., and Gosselin, J. (2010). J Immunol 185, 3620-3631.

Fitzgerald, K.A., McWhirter, S.M., Faia, K.L., Rowe, D.C., Latz, E., et al. (2003). Nat Immunol 4, 491-496.

Gariano, G.R., Dell'Oste, V., Bronzini, M., Gatti, D., Luganini, A., et al. (2012). PLoS 
Pathog 8, e1002498.

Hemmi, H., Takeuchi, O., Kawai, T., Kaisho, T., Sato, S., et al. (2000). Nature 408, 740-745.

Hochrein, H., Schlatter, B., O'Keeffe, M., Wagner, C., Schmitz, F., et al. (2004). Proc Natl Acad Sci U S A 101, 11416-11421.

Honda, K., Yanai, H., Mizutani, T., Negishi, H., Shimada, N., et al. (2004). Proc Natl Acad Sci U S A 101, 15416-15421.

Hornung, V., Ablasser, A., Charrel-Dennis, M., Bauernfeind, F., Horvath, G., et al. (2009). Nature 458, 514-518.

Ishii, K.J., and Akira, S. (2006). Trends Immunol 27, 525-532.

Ishii, K.J., Kawagoe, T., Koyama, S., Matsui, K., Kumar, H., et al. (2008). Nature 451, 725-729.

Ishikawa, H., and Barber, G.N. (2008). Nature 455, 674-678.

Ishikawa, H., Ma, Z., and Barber, G.N. (2009). Nature 461, 788-792.

Kato, H., Takeuchi, O., Sato, S., Yoneyama, M., Yamamoto, M., et al. (2006). Nature 441, 101-105.

Kawai, T., and Akira, S. (2009). Int Immunol 21, 317-337.

Kawai, T., and Akira, S. (2011). Immunity 34, 637-650.

Kawai, T., Takahashi, K., Sato, S., Coban, C., Kumar, H., et al. (2005). Nat Immunol 6, 981-988.

Kerur, N., Veettil, M.V., Sharma-Walia, N., Bottero, V., Sadagopan, S., et al. (2011). Cell Host Microbe 9, 363-375.

Konno, H., Yamamoto, T., Yamazaki, K., Gohda, J., Akiyama, T., et al. (2009). PloS One 4, e5674.

Krug, A., French, A.R., Barchet, W., Fischer, J.A., Dzionek, A., et al. (2004). Immunity 21, 107-119.

Kumar, H., Kawai, T., and Akira, S. (2011). Int Rev Immunol 30, 16-34.

Kumar, H., Kawai, T., Kato, H., Sato, S., Takahashi, K., et al. (2006). J Exp Med 203, 1795-1803.

Kumar, S., Ingle, H., Prasad, D.V., and Kumar, H. (2012). Crit Rev Microbiol. (In Press).

Landolfo, S., Gariglio, M., Gribaudo, G., and Lembo, D. (1998). Biochimie 80, 721-728.
Latz, E., Schoenemeyer, A., Visintin, A., Fitzgerald, K.A., Monks, B.G., et al. (2004). Nat Immunol 5, 190-198.

Li, Y., Chen, R., Zhou, Q., Xu, Z., Li, C., et al. (2012). Proc Natl Acad Sci U S A 109, 11770-11775.

Liu, Y.T., and Yin, H.L. (1998). J Biol Chem 273, 7920-7927.

Loo, Y.M., and Gale, M., Jr. (2011). Immunity 34, 680-692.

Medzhitov, R. (2007). Nature 449, 819-826.

Meylan, E., Curran, J., Hofmann, K., Moradpour, D., Binder, M., et al. (2005). Nature 437, 1167-1172.

Mok, B.W., Song, W., Wang, P., Tai, H., Chen, Y., et al. (2012). J Virol 86, 12695-12707.

Muruve, D.A., Petrilli, V., Zaiss, A.K., White, L.R., Clark, S.A., et al. (2008). Nature 452, 103-107.

O'Neill, L.A., and Bowie, A.G. (2010). Curr Biol 20, R328-333.

Orzalli, M.H., Deluca, N.A., and Knipe, D.M. (2012). Proc Natl Acad Sci U S A 109, E3008-E3017.

Ouyang, S., Song, X., Wang, Y., Ru, H., Shaw, N., et al. (2012). Immunity 36, 1073-1086.

Palm, N.W., and Medzhitov, R. (2009). Immunol Rev 227, 221-233.

Parvatiyar, K., Zhang, Z., Teles, R.M., Ouyang, S., Jiang, Y., et al. (2012). Nat Immunol. (In Press).

Petrilli, V., Dostert, C., Muruve, D.A., and Tschopp, J. (2007). Curr Opin Immunol 19, 615-622.

Rathinam, V.A., Jiang, Z., Waggoner, S.N., Sharma, S., Cole, L.E., et al. (2010). Nat Immunol 11, 395-402.

Ronfani, L., Ferraguti, M., Croci, L., Ovitt, C.E., Scholer, H.R., et al. (2001). Development 128, 1265-1273.

Sasai, M., Linehan, M.M., and Iwasaki, A. (2010). Science 329, 1530-1534.

Sauer, J.D., Sotelo-Troha, K., von Moltke, J., Monroe, K.M., Rae, C.S., et al. (2011). Infect Immun 79, 688-694.

Seth, R.B., Sun, L., Ea, C.K., and Chen, Z.J. (2005). Cell 122, 669-682.

Shang, G., Zhu, D., Li, N., Zhang, J., Zhu, C., et al. (2012). Nat Struct Mol Biol 19,
725-727.

Sharma, S., tenOever, B.R., Grandvaux, N., Zhou, G.P., Lin, R., et al. (2003). Science 300, 1148-1151.

Shu, C., Yi, G., Watts, T., Kao, C.C., and Li, P. (2012). Nat Struct Mol Biol 19, 722-724.

Stein, S.C., and Falck-Pedersen, E. (2012). J Virol 86, 4527-4537.

Stetson, D.B., and Medzhitov, R. (2006). Immunity 24, 93-103.

Sun, Q., Sun, L., Liu, H.H., Chen, X., Seth, R.B., et al. (2006). Immunity 24, 633-642.

Takaoka, A., Wang, Z., Choi, M.K., Yanai, H., Negishi, H., et al. (2007). Nature 448, 501-505.

Unterholzner, L., Keating, S.E., Baran, M., Horan, K.A., Jensen, S.B., et al. (2010). Nat Immunol 11, 997-1004.

Upton, J.W., Kaiser, W.J., and Mocarski, E.S. (2012). Cell Host Microbe 11, 290-297.

Vaccari, T., Beltrame, M., Ferrari, S., and Bianchi, M.E. (1998). Hmg4, a new member of the Hmg1/2 gene family. Genomics 49 , 247-252.

Valentine, R., and Smith, G.L. (2010). J Gen Virol 91, 2221-2229.

Veeranki, S., and Choubey, D. (2012). Mol Immunol 49, 567-571.

Veeranki, S., Duan, X., Panchanathan, R., Liu, H., and Choubey, D. (2011). Plos one 6, e27040.

Wagner, H. (2004). Trends Immunol 25, 381-386.

Wenzel, M., Wunderlich, M., Besch, R., Poeck, H., Willms, S., et al. (2012). J Immunol 188, 394-403.

Xu, L.G., Wang, Y.Y., Han, K.J., Li, L.Y., Zhai, Z., and Shu, H.B. (2005). Mol Cell 19, 727-740.

Yanai, H., Ban, T., Wang, Z., Choi, M.K., Kawamura, T., et al. (2009). Nature 462, 99-103.

Yang, P., An, H., Liu, X., Wen, M., Zheng, Y., et al. (2010). Nat Immunol 11, 487-494.

Yin, Q., Tian, Y., Kabaleeswaran, V., Jiang, X., Tu, D., et al. (2012). Mol Cell 46, 735-745.

Zhang, Z., Yuan, B., Bao, M., Lu, N., Kim, T., et al. (2011). Nat Immunol 12, 959-965.

Zhong, B., Yang, Y., Li, S., Wang, Y.Y., Li, Y., et al. (2008). Immunity 29, 538-550. 